\title{
In-vitro mobility of the lumbar spine
}

\author{
R. C. HILTON, ${ }^{1}$ J. BALL, ${ }^{2}$ AND R. T. BENN ${ }^{3}$
}

From the ${ }^{1}$ Department of Rheumatology, Hope Hospital, Manchester, the ${ }^{2}$ Department of Rheumatolog University of Manchester, and the ${ }^{3}$ Department of Community Medicine, University of Manchester

SUMMARY As part of a systematic study of the spine between D11 and S1 the response to stress. has been assessed by measuring radiologically the total and segmental mobility of 103 specimenæ The method was shown to be reproducible to within $10 \%$ of total mobility. No significant sef difference was seen. Total mobility varied widely, but mean total mobility fell with age. All lumbar segments became less mobile up to the fifth decade. Between the fifth and the eighth decade the mobility of the fifth lumbar segment continued to fall, whereas other lumbar segments became morow mobile. The mobility of each segment also varied widely, but the mean segmental mobility feP progressively from L 5 to D11. However, this smooth pattern of movement was seen in only 16 cases mostly adolescents and young adults. Among the remainder, in which movement was irregularl distributed, there were 7 cases ( 4 aged less than 30 years) in which the mobility of at least 1 segment was more than 2 standard deviations above the mean. The cause of this hypermobility was not evident radiologically. It is suggested that segments with abnormally high mobility may be at risk. है

Lumbar spinal mobility was first studied by Weber and Weber (1836) on 3 post-mortem specimens. Since then most investigators have measured mobility clinically, with or without the aid of radiographs (Elward, 1939; Gianturco, 1944; Tanz, 1953; Allbrook, 1957; Israel, 1959; Jonck and Van Niekerk, 1961; Clayson et al., 1962; Lindahl, 1966; Loebl, 1967; Sturrock et al., 1973; Sweetman et al., 1974). Post-mortem studies of dorsolumbar mobility have been neglected despite the advantage that they facilitate analysis of mobility patterns and their relation to structural abnormalities.

The measurements of sagittal mobility described in this paper were obtained as part of a systemic study of the spine below $\mathrm{D} 9$.

\section{Material and methods}

The material consisted of 117 selected post-mortem specimens of the lower spine. Specimens were not removed from patients with known neoplasia or metabolic bone disease or if they had received longterm corticosteroids. Histological examination of an undecalcified bone block from the sacrum of each spine revealed 5 patients with unsuspected osteomalacia and 1 with mild secondary hyperparathyroidism. These patients were not included in the

Accepted for publication 7 August 1978

Correspondence to $\mathrm{Dr}$ R. C. Hilton, Rheumatic Diseases Unit, Hope Hospital, Eccles Old Road, Salford M6 8HD. study. Of the remaining 111,52 were females and 59 were males. The age range was 13 to 96 years Fifty-three patients were under 50 years and 58 were over 50 years of age. Most patients had died withi通 1 week of admission to hospital, and none had bee confined to bed for more than 1 month.

\section{MEASUREMENT OF SAGITTAL MOBILITY}

The method used was an adaptation of that dese cribed by Ball and Meijers (1964) for mobility $\vec{\rho}$ studies on the cervical spine. The lower spine? including the sacrum, was removed by dividing the ninth dorsal vertebra and the sacroiliac joints? Measurements were made at room temperature in a humid atmosphere. In most cases fresh specio mens were used. The remainder were stored at $4^{\circ} \mathrm{C}$ wrapped in several layers of gauze soaked in saline and brought to room temperature before measuring mobility. It has been shown that this storing pro cedure does not affect the mechanical properties (Kazarian, 1972). A steel pin was inserted into the anterior aspect of each vertebral body from D1\& to $\mathrm{S} 1$ inclusive. The sacrum was fixed in a clamf with the spine and pins lying in the horizontal plane about $5 \mathrm{~cm}$ above the surface of a table. A line attached to D9 was led anteriorly over a pulley to at cloth bag containing a $2 \mathrm{~kg}$ load. This load waș selected because preliminary experiments had showr that with greater loads there was a risk of fracture, especially in older specimens. In a few younger 
cases $4 \mathrm{~kg}$ was also used, and it was found that this increased total mobility by up to $25 \%$.

When maximum flexion had been achieved the specimen was maintained under load for approximately 10 minutes. This time lapse was selected because experiments on 2 spines had shown that the increase in total mobility due to creep was most marked in the first 10 minutes $(8 \%$ and $6.4 \%)$ and that any subsequent increase was within experimental error (not more than $1^{\circ}$ ). Creep had no discernible effect on the pattern of mobility. A radiograph was then taken with the tube $110 \mathrm{~cm}$ above the table and centred over the mid point between D10 and S1. After the pulley and bar were transferred to the posterior side the procedure described above was repeated to obtain an $x$-ray in maximum extension.

The two radiographs were used to measure angular movement about each of the 8 discs between D10 and S1. With the pin in S1 being taken for reference, the difference between the angle subtended by the pin in L5 in flexion and extension gave the angular movement of L5 on S1. The angular movement of L4 with reference to S1 was then calculated, and the difference between this angle and that calculated for L5 gave the movement of L4 on L5. This procedure was repeated for each disc level.

The reproducibility of the method was tested by repeated measurements on 3 specimens and found to be within $10 \%$ of total mobility, with no change in the segmental distribution (Table 1).

\section{Results}

Among the 111 cases 5 unsuspected cases of primary instability and 3 unsuspected cases of spondylolisthesis were found. These were excluded, thus leaving 103 cases for analysis. There were 25 males
Table 1 Triplicate measurements of mobility

\begin{tabular}{|c|c|c|c|c|c|c|}
\hline \multicolumn{4}{|c|}{ Specimen 1 (F37) } & \multicolumn{3}{|c|}{ Specimen 2 (M53) } \\
\hline $\begin{array}{l}\text { L5 } \\
\text { L4 } \\
\text { L3 } \\
\text { L2 } \\
\text { L1 } \\
\text { D12 } \\
\text { D11 } \\
\text { D10 }\end{array}$ & $\begin{array}{c}7 \\
14 \cdot 5 \\
9 \cdot 5 \\
7 \\
10 \\
4 \\
3 \cdot 5 \\
2\end{array}$ & $\begin{array}{l}7 \\
14 \\
10 \\
8 \cdot 5 \\
9 \cdot 5 \\
3 \cdot 5 \\
3 \cdot 5 \\
1 \cdot 5\end{array}$ & $\begin{array}{l}7 \cdot 5 \\
14 \\
10 \\
8 \\
10 \\
4 \cdot 5 \\
3 \\
2 \cdot 5\end{array}$ & $\begin{array}{l}18 \\
9 \\
6 \cdot 5 \\
6 \cdot 5 \\
4 \\
2 \cdot 5 \\
1 \\
0 \cdot 5\end{array}$ & $\begin{array}{l}18 \\
9 \cdot 5 \\
6 \cdot 5 \\
7 \\
4 \cdot 5 \\
2 \cdot 5 \\
1 \\
0 \cdot 5\end{array}$ & $\begin{array}{l}18 \cdot 5 \\
9 \\
7 \\
6 \cdot 5 \\
4 \cdot 5 \\
2 \\
1 \cdot 5 \\
0 \cdot 5\end{array}$ \\
\hline Total & $57 \cdot 5$ & $57 \cdot 5$ & $59 \cdot 5$ & 48 & $49 \cdot 5$ & $49 \cdot 5$ \\
\hline
\end{tabular}

and 23 females aged under 50 years of age and 29 males and 26 females aged 50 years and over.

\section{CHANGES IN MOBILITY WITH AGE}

At all ages there is a marked variation in total mobility, though the mean falls with age by about $25 \%$ between those aged up to 30 and those aged over 70 years (Fig. 1). No sex difference in these variables was seen.

The mean mobility of each lumbar segment is shown for males in Fig. 2. Segmental mobility falls with age up to the fifth decade. Thereafter the mobility of the fifth lumbar segment continues to fall to the eighth decade whereas that of L4, L3, L2, and L1 tends to increase. The results in females were similar, though the fall in mobility at L5 was less uniform. The fall in mobility at L5 in the elderly is shown most strikingly when the mean mobility at each disc level in the age groups less than 50 and $50+$ years are compared (Fig. 3). It can be seen that in the older age group reduced mobility is virtually confined to the L5 segment, A study of the grades of disc narrowing and osteophyte formation at each disc level (to be reported elsewhere) showed that this fall in mobility at L5 in males and females could not be attributed to either of these factors. More disc narrowing occurred at L5 than at other segments,

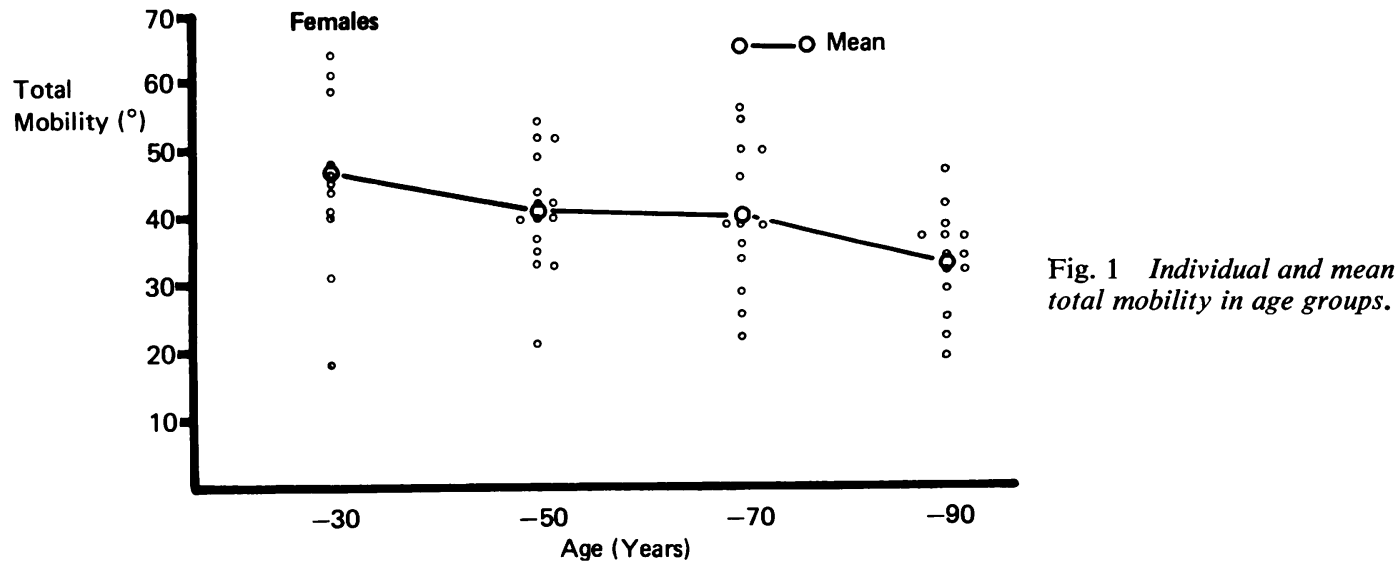

Fig. 1 Individual and mean 


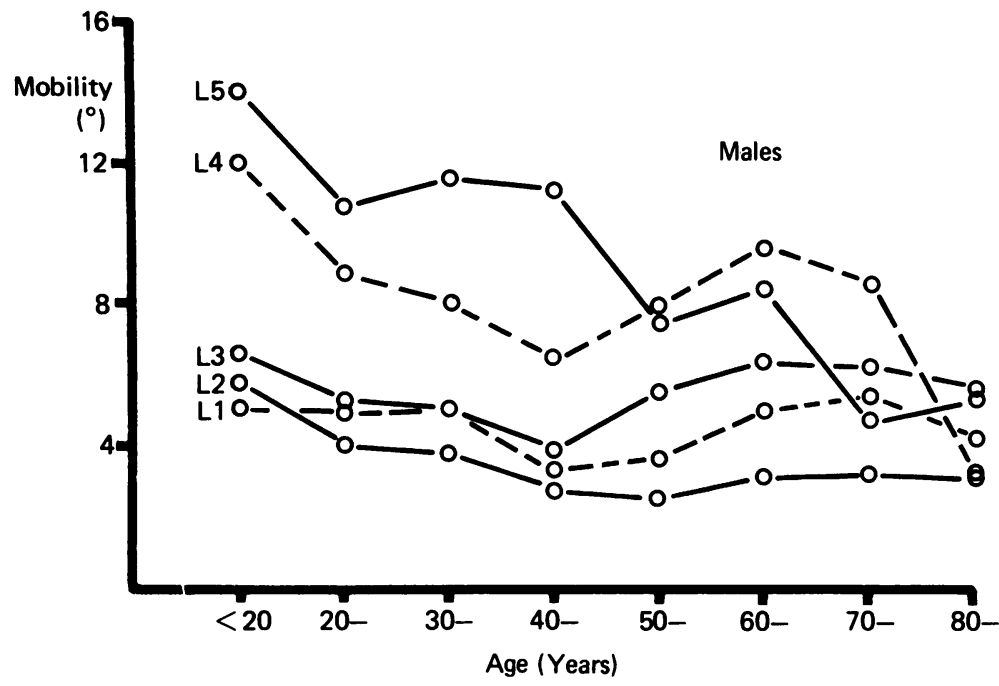

Fig. 2 Mean mobility by age and disc level.

but even when cases with disc narrowing at L5 are excluded, the fall in mobility at L5 is still present (Fig. 3).

\section{MOBILITY PATTERNS}

In both sexes there is a considerable scatter of
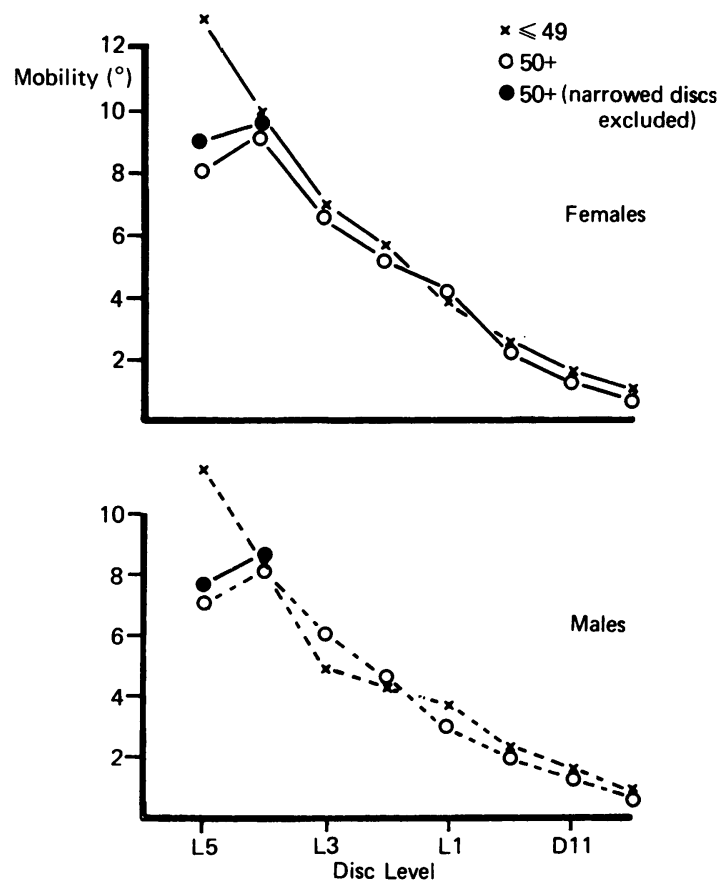

Fig. 3 Mean mobility by disc level in age groups $₹ 49$ and $50+$ years. mobility values at each disc level, but the mean mobility falls progressively from L5 (Fig. 4). How-1 ever, this smooth fall in mean mobility is misleading since in the majority of individuals mobility is to a varying extent irregularly distributed. Indeed, only 9 females and 7 males conform to this mean mobilit pattern. Of these 16,13 are aged less than 50 yearso

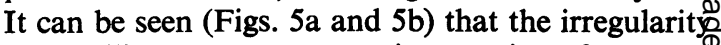
in mobility patterns occurs irrespective of age and sex and can be due to either an abnormal reduction $\vec{b}$ or an increase in mobility at any disc level or a combination of these (Fig. 6).

In both sexes approximately $25 \%$ of mobility. values at each segment are greater than plus of minus one standard deviation from the mean anc greater than 2 standard deviations between 1 an 9 $10 \%$ (Fig. 4). In discs with mobility greater tham 2 standard deviations below the mean only $45 \%$ showed radiological evidence of disc degeneration In none of the examples shown in Fig. 5a was there disc degeneration. In the 7 cases with isolate segmental mobility greater than 2 standard devia tions above the mean (Fig. 5b) 4 were aged less tharo 30 years and were radiologically normal. The other 3 showed only minimal radiological evidence of dis\& degeneration of the affected discs. It can be seen (Fig. 5b) that the abnormal segmental mobility in alf 7 is not due to reduced mobility at other levels.

\section{Discussion}

Clinical assessments of total sagittal mobility im healthy individuals have yielded variable results (Israel, 1959; Lindahl, 1966; Loebl, 1967; Sturrock ${ }^{2}$ et al., 1973; Sweetman et al., 1974) partly because of 


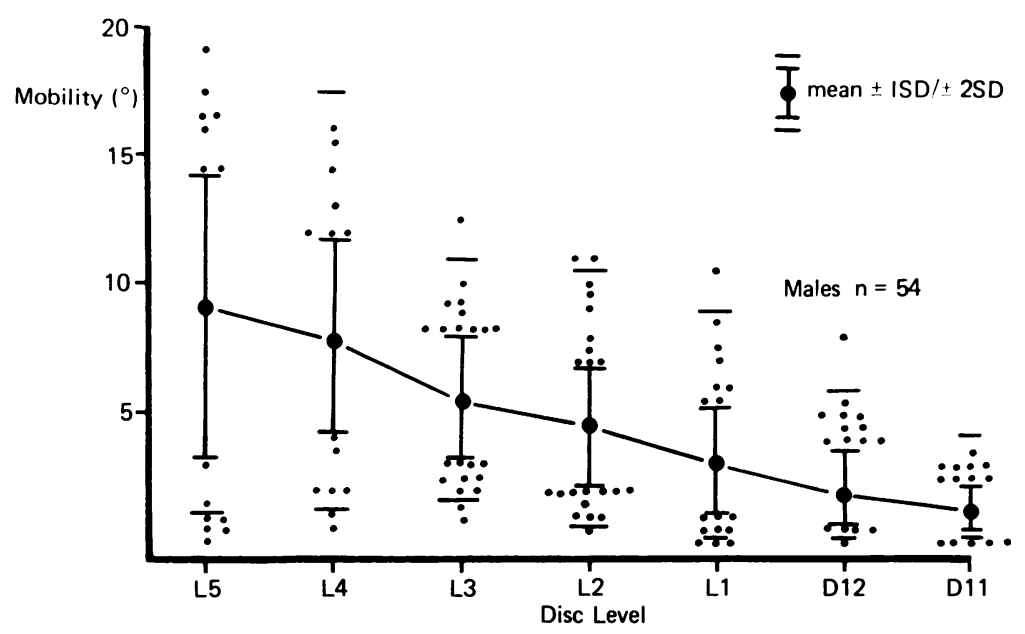

Fig. 4 Mean mobility and discs with mobility $\pm 1 S D$ and $\pm 2 S D$ at each disc level.
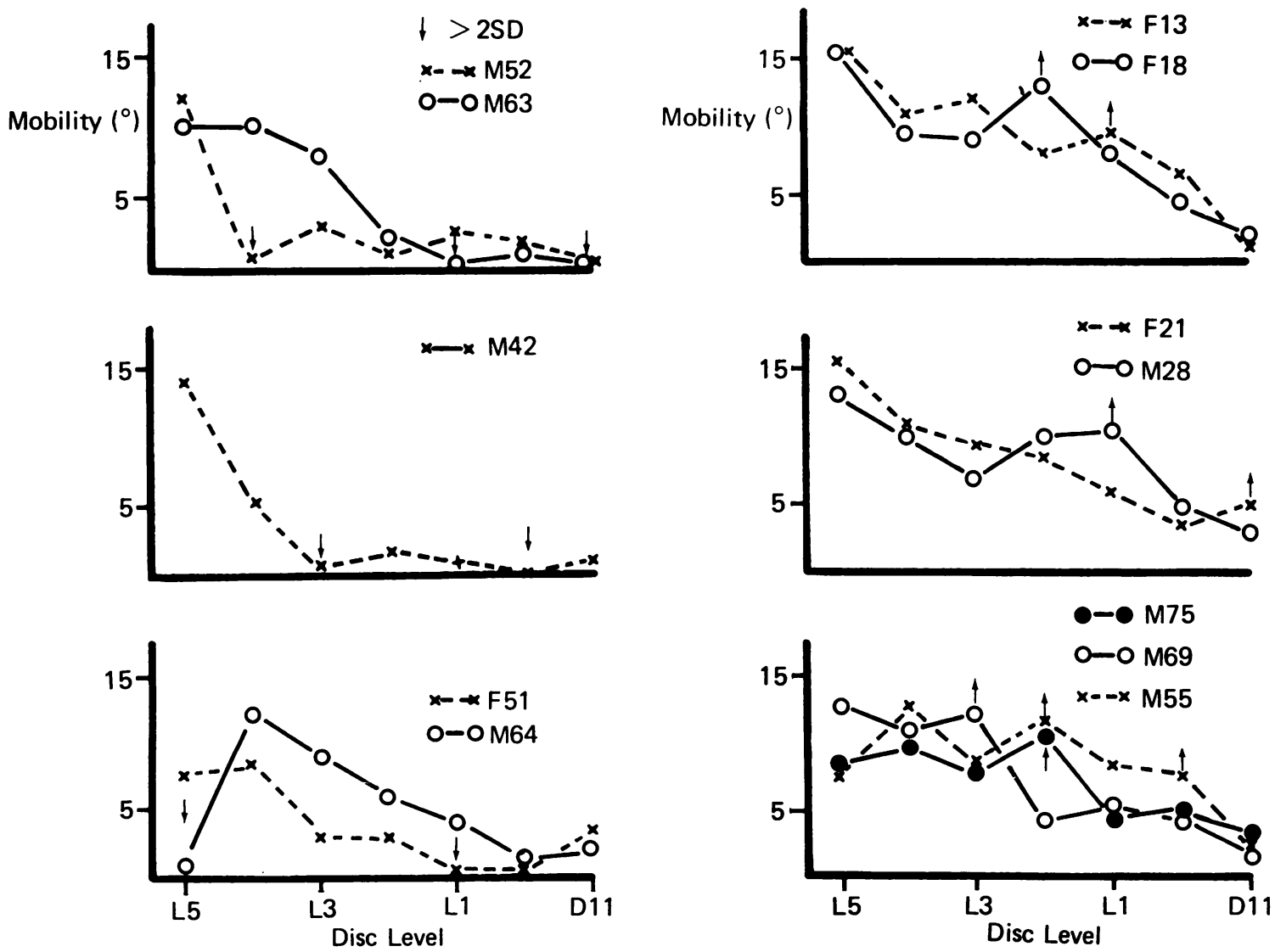

Fig. 5 Irregular spinal mobility patterns: (a) due to segmental reduction in mobility; ((b) due to segmental increase in mobility. 


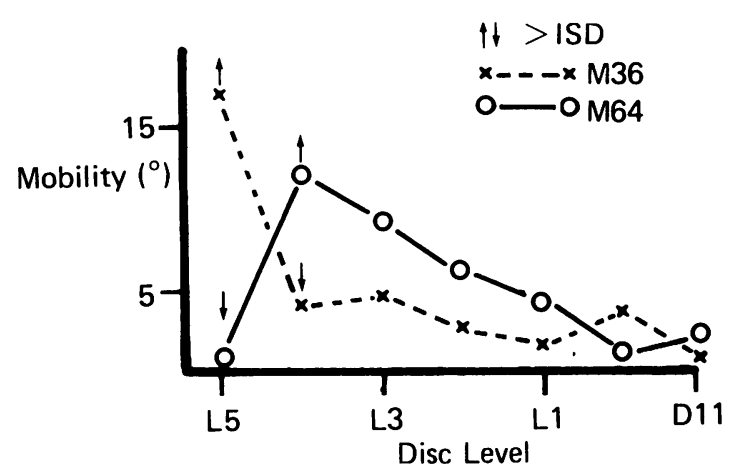

Fig. 6 Irregular mobility patterns due to combined reduction and increased mobility.

differences in the consistency and accuracy of the methods used (Reynolds, 1975). Movement between individual discs can be measured only by radiographs (Elward, 1939; Gianturco, 1944; Tanz, 1953; Allbrook, 1957; Jonck and Van Niekerk, 1961 ; Clayson et al., 1962), but such studies have been infrequent.

In clinical studies total mobility and its distribution may of course be influenced by the presence or absence of pain and the effectiveness of muscular activity. Pathological studies are not subject to these constraints and hence may provide a more accurate estimate of the underlying mechanical stability as determined by ligamentous and bony structures.

The simple method adopted in the present study was shown to yield reproducible results for sagittal mobility of the whole specimen (D11-S1) and its constituent segments. No attempt was made to preload the spine (equivalent to the erect posture), and the findings might be criticised on this account. According to Panjabi et al. (1977) preloading would tend to increase sagittal mobility, but there were no grounds for assuming that the segmental distribution of mobility would be altered, though this cannot be excluded. In fact the overall spinal mobility between D11 and L5 observed in the present in vitro studies was found to be of a similar order to that reported for this region in healthy recumbent subjects by Tanz (1953) using radiological methods, but some $30 \%$ less than that reported by Loebl (1967) for each decade using a goniometer and measuring flexion in the sitting position and extension with the patient prone. The higher mobility reported by Loebl (1967) could have been achieved in the present experiment by doubling the load, but for practical reasons (see above) this degree of loading could not be used. At all ages in both sexes there is a wide range of total mobility. Mean total mobility in adult life is similar in males and females, and in both sexes falls by about $25 \%$ between those aged up to 30 and those aged over 70 years.

Surprisingly, almost all the loss of mobility in the elderly occurs at L5 in both sexes and is accompanie $\$$ by an apparent compensatory increase in mobilit above L4. This compensating increase in mobility which has not previously been reported, could meap that the dorsolumbar junction becomes less resistanth to stress in the middle aged and elderly. A dis proportionate loss of mobility at L5 was also reported in the clinical studies of Tanz $(1950,1953)$, who lik $\overrightarrow{\mathrm{e}}$ ourselves could not explain this on the basis of dis degeneration.

Mean segmental mobility falls progressively fror L5. However, there is a wide scatter of mobility at each disc level. This finding is not accounted for bs high and low mobility spines but reflects irregulario ties in the distribution of mobility in individuat spines. Thus, spines conforming to the regular pattern of mobility described by the mean values are rarely found in adults. The irregular distribution of mobility is due to either decreased or increase $\mathscr{f}^{4}$ mobility in 1 or more segments or a combination of these abnormalities. The irregularities are oftepo unpredictable from straight $x$-rays and do not necessarily reflect gross pathology, since they car occur in relatively healthy young specimens. If about $7 \%$ of our specimens one or more segmenty had a sagittal mobility greater than two standar deviations above the mean. This type of abnormal movement was not explicable on the basis of reduce? mobility at other levels. It was found at all levels, except L4 and L5. In 4 of the 7 cases it occurred if healthy spines aged less than 30 years. The nature of the 'hypermobility' is obscure, but it cannot bE attributed to primary instability in the sense og Morgan and King (1957), since such cases have beef excluded radiologically, and, unlike primary inê stability, there was no predilection to affect the $\mathrm{L}_{4} \xi$ L5 disc. However, it seems reasonable to assume that such hypermobile segments may be at risk to mechanical stress. Clinical methods designed to detect such segments may be of some value in tha study of low back pain.

\section{References}

Allbrook D. (1957), Movements of the lumbar spinal Journal of Bone and Joint Surgery, 39B, 339-345.

Ball, J., and Meijers, K. A. E. (1964). On cervical mobility Annals of the Rheumatic Diseases, 23, 429-438.

Clayson, S. J., Newman, I. M., Debevec, D. F., Anger, R. W.; Showlund, H. V., and Kottke, F. J. (1962). Evaluation of mobility of hip and lumbar vertebrae of normal young women. Archives of Physical Medicine and Rehabilitation 43, 1-8.

Elward, J. F. (1939). Motion in the vertebral column American Journal of Roentgenology and Radium Therapy 42, 91-99. 
Gianturco, C. (1944). A roentgen analysis of the motion of the lower lumbar vertebrae in normal individuals and in patients with low back pain. American Journal of Roentgenology and Radium Therapy, 52, 261-268.

Israel, M. (1959). A quantitative method of estimating flexion and extension of the spine-a preliminary report. Military Medicine, 124, 181-186.

Jonck, L. M., and Van Niekerk, J. M. (1961). A roentgenological study of the motion of the lumbar spine of the Bantu. South African Journal of Laboratory and Clinical Medicine, 2, 67-71.

Kazarian, L. E. (1972). Dynamic response characteristics of the human vertebral column. Acta Orthopaedica Scandinavica, Suppl. 146.

Lindahl, O. (1966). Determination of the sagittal mobility of the lumbar spine. Acta Orthopaedica Scandinavica, 37, 241-254.

Loebl, W. Y. (1967). Measurement of spinal posture and range of spinal movement. Annals of Physical Medicine, 9, 103-110.

Morgan, F. P., and King, T. (1957). Primary instability of lumbar vertebrae as a common cause of low back pain. Journal of Bone and Joint Surgery, 39B, 6-22.
Panjabi, M. M., Krag, M. H., White III A. A., and Southwick, W. O. (1977). Effects of preload on load displacement curves of the lumbar spine. Orthopaedic Clinics of America, 8, 181-192.

Reynolds, P. M. C. (1975). Measurement of spinal mobility: a comparison of three methods. Rheumatology and Rehabilitation, 14, 180-185.

Sturrock, R. D., Wojtulewski, J. A., and Dudley Hart, F. (1973). Spondylometry in a normal population and in ankylosing spondylitis. Rheumatology and Rehabiliation, 12, 135-142.

Sweetman, B. J., Anderson, A. D., and Dalton, E. R. (1974). The relationships between little finger mobility, lumbar mobility, straight-leg raising, and low back pain. Rheumatology and Rehabilitation, 13, 161-166.

Tanz, S. S. (1950). To-and-fro motion range at the fourth and fifth lumbar interspaces. Journal of the Mount Sinai Hospital, 16, 303-307.

Tanz, S. S. (1953). Motion of the lumbar spine. American Journal of Roentgenology and Radium Therapy, 39, 399-410.

Weber, W., and Weber, E. H. (1836). Mechanik der Menschlichen Gewerkzeuge, p. 109-113. Göttingen. 